\title{
THE MARKOFF SPECTRUM OF AN ALGEBRAIC NUMBER FIELD
}

\author{
A. C. WOODS \\ To Kurt Mahler on his seventy-fifth birthday
}

(Received 4 January 1978)

Communicated by J. H. Coates

\begin{abstract}
The Markoff spectrum of an algebraic number field is defined and it is proved that the spectrum of $Q(\sqrt{ } 5)$ is not discrete.
\end{abstract}

Subject classification (Amer. Math. Soc. (MOS) 1970): 12 A 25

Let $K$ be a finite extension of the rational numbers $\mathbf{Q}$ find let $M$ be a full module of $K$. Further, denote by $n(M)$ the least value of $|N(m)|$ as $m$ runs over all nonzero elements of $M$ and by $D(M)$ the discriminant of $M$. Put

$$
\mu(M)=n(M) / \sqrt{ }(|D(M)|) .
$$

The number $\mu(M)$ is an invariant of the similarity class of modules containing $M$. In analogy to the Markoff spectrum of real indefinite quadratic forms the set of such numbers $\mu(M)$ is called the Markoff spectrum of $K$. The first question concerning this set of numbers is whether it possesses any finite limit points. Here we prove the following:

THEOREM. The Markoff spectrum of $\mathbf{Q}(\sqrt{5})$ has at least one limit point.

Proof. Let $u_{0}=1, u_{1}=1$ and, in general, $u_{n+2}=u_{n+1}+u_{n}, n \geqslant 0$, be the Fibonacci numbers. Denote by $L_{n}$ the sublattice of $L$ of basis

$$
\left(u_{n}, u_{n}\right),\left(\left(2 u_{n-1}+u_{n}\right) w,\left(2 u_{n-1}+u_{n}\right) w^{\prime}\right) .
$$


It suffices to prove $m\left(L_{n}\right)=u_{n}^{2}$, for $n \geqslant 3$, as in this case, since

it follows that

$$
d\left(L_{n}\right)=u_{n}\left(2 u_{n-1}+u_{n}\right) \sqrt{ } 5
$$

$$
\mu\left(L_{n}\right)=1 /\left(2\left(u_{n-1} / u_{n}\right)+1\right) \sqrt{ } 5>1 / 3 \sqrt{ } 5 .
$$

Since the ratios $u_{n-1} / u_{n}$ are all distinct, the theorem follows.

It remains to show $m\left(L_{n}\right)=u_{n}^{2}$ for $n \geqslant 3$. In fact this is also true when $n=1,2$ but we do not need this.

The values taken by the quadratic form

$$
\begin{aligned}
F_{n}(x, y) & =\left(u_{n} x+w\left(2 u_{n-1}+u_{n}\right) y\right)\left(u_{n} x+w^{\prime}\left(2 u_{n-1}+u_{n}\right) y\right) \\
& =u_{n}^{2} x^{2}+\left(u_{n}^{2}+2 u_{n-1} u_{n}\right) x y-\left(2 u_{n-1}+u_{n}\right)^{2} y^{2},
\end{aligned}
$$

as $(x, y)$ ranges over all pairs of rational integers other than $(0,0)$, are precisely the values of $x_{1} x_{2}$ as $\left(x_{1}, x_{2}\right)$ ranges over all points of $L_{n}$ other than 0 . Hence it suffices to show that the minimal value of $\left|F_{n}(x, y)\right|$ is $u_{n}^{2}$ when $(x, y)$ ranges over all pairs of rational integers other than $(0,0)$. We use the classical theory of reduction of binary indefinite quadratic forms to complete the proof, see for example Dickson (1930).

The standard notation for continued fractions is employed, so that $\left(a_{1}, \ldots, a_{m}\right)$ denotes the simple continued fraction

$$
a_{1}+1 /\left(a_{2}+1 /\left(a_{3}+\ldots+1 / a_{n}\right) \ldots\right)
$$

of finite length, whereas $\left(a_{1}, \overline{a_{2}, \ldots, a_{n}}\right)$ denotes the simple continued fraction of infinite length obtained when the block $a_{2}, \ldots, a_{n}$ is repeated a countable number of times.

LEMMA. For $n \geqslant 1$, if $\theta=(1, \overline{2,1, \ldots, 1,3,1, \ldots, 1,4})$ then $\theta$ is a root of $F_{n+2}(x, 1)$.

ProOF. All rational numbers appearing in this proof will be in lowest terms.

For $n \geqslant 1$, we have $(\stackrel{+, \ldots, 1}{1, \ldots,})=u_{n} / u_{n-1}$, and so

$$
\begin{aligned}
& (\overleftarrow{1, \ldots,} \overrightarrow{1}, 4)=\left(4 u_{n}+u_{n-1}\right) /\left(u_{n}+3 u_{n-1}\right) \\
& (\stackrel{\leftarrow-1, \ldots,}{1}, \ldots)=\left(u_{n}+2 u_{n-1}\right) /\left(2 u_{n}-u_{n-1}\right) \text {, }
\end{aligned}
$$

and therefore also

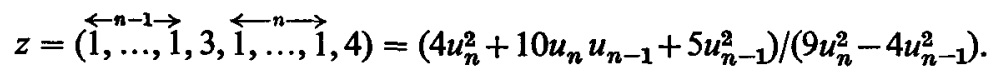

Hence

$$
\begin{aligned}
(2, z) & =\left(17 u_{n}^{2}+20 u_{n} u_{n-1}+6 u_{n-1}^{2}\right) /\left(4 u_{n}^{2}+10 u_{n} u_{n-1}+5 u_{n-1}^{2}\right) \\
& =\left(3 u_{n+2}^{2}+2 u_{n+2} u_{n+1}+u_{n+1}^{2}\right) /\left(4 u_{n+1}^{2}+2 u_{n+1} u_{n+2}-u_{n+2}^{2}\right)
\end{aligned}
$$


Next,

and so,

$$
\begin{aligned}
w & =(\stackrel{\leftrightarrow-1, \ldots, 1}{1}, 3, \stackrel{\leftrightarrow}{1}, \ldots, \overrightarrow{1}) \\
& =\left(u_{n}^{2}+2 u_{n} u_{n-1}+u_{n-1}^{2}\right) /\left(2 u_{n}^{2}-u_{n-1}^{2}\right)
\end{aligned}
$$

$$
(2, w)=u_{n+2}^{2} / u_{n+1}^{2}
$$

Hence, if $\psi=(2, z, \psi)$ then $\psi$ satisfies the equation

$$
\psi=\frac{\left(3 u_{n+2}^{2}+2 u_{n+2} u_{n+1}+u_{n+1}^{2}\right) \psi+u_{n+2}^{2}}{\left(4 u_{n+1}^{2}+2 u_{n+1} u_{n+2}-u_{n+2}^{2}\right) \psi+u_{n+1}^{2}}
$$

and therefore also $\theta=1+1 / \psi$ satisfies $F_{n+2}(\theta, 1)=0$, which proves the lemma.

It now follows that the ordered set of integers

$$
2,1, \ldots, 1,3,1, \ldots, 1,4
$$

is a period of the form $F_{n+2}(x, y)$. Hence, applying the classical theory of reduction of indefinite binary quadratic forms, see for example Dickson (1930), it is easy to see that the required minimum value of $F_{n+2}(x, y)$ is attained for $(x, y)=(p, q)$ where $p$ and $q$ are relatively prime integers for which

$$
p / q=(1,2,1, \ldots, 1,3,1, \ldots, 1) .
$$

As $p / q=(1,2, w)=\left(u_{n+2}^{2}+u_{n+1}^{2}\right) / u_{n+2}^{2}$, so the required minimum value is

$$
\left|F_{n+2}\left(u_{n+2}^{2}+u_{n+1}^{2}, u_{n+2}^{2}\right)\right|=u_{n+2}^{2}
$$

since

$$
u_{n+2}^{2}+2 u_{n+2} u_{n+1}-u_{n+1}^{2}=(-1)^{n} \text {. }
$$

This completes the proof of the theorem.

\section{References}

J. W. S. Cassels and H. P. F. Swinnerton-Dyer (1955), "On the product of three homogeneous linear forms and indefinite ternary quadratic forms", Phil. Trans. Royal Soc. London A 248, 73-96.

L. G. Dickson (1930), Studies in the Theory of Numbers (University of Chicago Press).

H. P. F. Swinnerton-Dyer (1971), "On the product of three homogeneous linear forms", Acta Arith. 18, 371-385.

The Ohio State University

Columbus, Ohio

USA 